ISSN 0103-9954

\title{
LEVANTAMENTO POPULACIONAL E ANÁLISE FAUNÍSTICA DE LEPIDOPTERA EM Eucalyptus spp. NO MUNICÍPIO DE PINHEIRO MACHADO, RS
}

\author{
SURVEY POPULATION AND FAUNAANALYSIS OF LEPIDOPTERA IN Eucalyptus spp. \\ IN THE MUNICIPALITY OF PINHEIRO MACHADO, RS
}

Oderlei Bernardi ${ }^{1}$ Mauro Silveira Garcia ${ }^{2}$ Eduardo José Ely e Silva ${ }^{3}$

Luiza Cristiane Fialho Zazycki ${ }^{4}$ Daniel Bernardi ${ }^{5}$ Élder Finkenauer ${ }^{6}$

\begin{abstract}
RESUMO
O trabalho teve como objetivo coletar, identificar e caracterizar faunisticamente os lepidópteros associados a um plantio de Eucalyptus spp., no município de Pinheiro Machado, RS. No período de outubro de 2005 a outubro de 2007, a cada 15 dias, foram realizadas coletas de insetos com três armadilhas luminosas instaladas no interior do florestamento. Após procedimentos de triagem, transfixação e montagem os lepidópteros foram identificados comparando-os a coleções entomológicas e com uso de literatura especializada. Foram coletados 2.020 indivíduos, distribuídos em 14 famílias, 106 gêneros e 220 espécies. As famílias com maior número de espécies coletadas foram Noctuidae (59), Geometridae (30), Arctiidae (28) e Saturniidae (14). De acordo com a classificação faunística a maioria das espécies foi considerada não dominante, pouco frequente, rara e acidental. Dentre as espécies identificadas, foram observadas algumas, cujas lagartas são desfolhadoras do eucalipto; Oxydia agliata (Geometridae), Sarsina violascens (Lymantriidae), Automeris illustris, Eacles imperialis magnifica e Lonomia obliqua (Saturniidae), as quais necessitam ter suas populações monitoradas.
\end{abstract}

Palavras-chave: eucalipto; lepidópteros; armadilha luminosa; entomologia florestal.

\begin{abstract}
The objective of this research was to collect, to study and to characterize the fauna of lepidopterous associated with Eucalyptus spp., plantation in the Municipality of Pinheiro Machado, in RS state. In the period of October 2005 to October 2007, every 15 days, collections of insects were accomplished with three light traps. After selection and transfixion procedures, the lepidopterous were identified based on entomological collections and specialized literature. Two thousand and twenty individuals belonging to 14 families, 106 genera and 220 species were collected. The families with the highest number of species collected were: Noctuidae (59), Geometridae (30), Arctiidae (28) and Saturniidae (14). According to the fauna classification the most species were considered not dominant, uncommon, rare and accidental. Among the species identified, there were some whose larvae are defoliators of the eucalypts: Oxydia agliata,
\end{abstract}

1. Engenheiro Agrônomo, Doutorando em Entomologia, Escola Superior de Agricultura "Luiz de Queiroz", Universidade de São Paulo, Av. Pádua Dias 11, Caixa Postal 9, CEP 13418-900, Piracicaba (SP). oderleibernardi@yahoo.com.br

2. Engenheiro Agrônomo, Dr., Professor do Departamento de Fitossanidade, Faculdade de Agronomia Eliseu Maciel, Universidade Federal de Pelotas, Caixa Postal 354, CEP 96010-900, Pelotas (RS). garciasmauro@yahoo.com.br

3. Engenheiro Agrônomo, Dr., Taxonomista, Departamento de Fitossanidade, Faculdade de Agronomia Eliseu Maciel, Universidade Federal de Pelotas, Caixa Postal 354, CEP 96010-900, Pelotas (RS). eduelysilva@uol.com.br

4. Engenheira Agrônoma, Mestranda em Entomologia, Departamento de Fitossanidade, Faculdade de Agronomia Eliseu Maciel, Universidade Federal de Pelotas, Caixa Postal 354, CEP 96010-900, Pelotas (RS). luizazazycki@gmail.com

5. Engenheiro Agrônomo, Mestrando em Entomologia, Departamento de Fitossanidade, Faculdade de Agronomia Eliseu Maciel, Universidade Federal de Pelotas, Caixa Postal 354, CEP 96010-900, Pelotas (RS). dbernardi2004@yahoo.com.br

6. Técnico Agrícola, Votorantim Celulose \& Papel S/A, Unidade Extremo Sul, BR 116, Km 532, CEP 96160-000, Capão do Leão (RS). elder.finkenauer@vcp.com.br

Recebido para publicação em 15/04/2010 e aceito em 4/11/2010 
(Geometridae), Sarsina violascens (Lymantriidae), Automeris illustris, Eacles imperialis magnifica and Lonomia obliqua (Saturniidae), which needs to have their populations monitored.

Keywords: eucalypt; lepidopterous; light trap; forest entomology.

\section{INTRODUÇ̃̃O}

Nos últimos anos, o Rio Grande do Sul tem sido alvo de uma série de investimentos florestais, especialmente os que se referem ao cultivo de eucalipto. Como em qualquer outra plantação homogênea, os plantios florestais reduzem a diversidade de vegetação e, consequentemente, tornam o ambiente menos estável e com menor capacidade de absorver distúrbios (RISCH et al., 1983). Diante disso, é provável que paralelamente a expansão da eucaliptocultura aumente também os problemas entomológicos.

Segundo Santos et al. (1982), após as formigas cortadeiras, os lepidópteros desfolhadores constituem o grupo de insetos de maior importância à eucaliptocultura nacional, por conter espécies que ocorrem em surto, causando grande desfolha, em diversos plantios dessa cultura. No entanto, os lepidópteros também são considerados importantes bioindicadores de qualidade ambiental por atuarem nos ecossistemas florestais desempenhando funções de desfolhadores, decompositores, presas ou hospedeiros, estando a sua diversidade relacionada à ciclagem de nutrientes, dinâmica populacional de plantas e à relação predador/presa de um ecossistema (SILVEIRA NETO et al., 1995).

Alguns dos estudos de levantamento populacional de lepidópteros associados a cultivos florestais têm sido executados de uma forma sistemática, por meio do recolhimento de ovos, larvas e adultos, levados ao laboratório, identificados ou criados para posterior identificação (BERTI FILHO, 1981; ZANUNCIO et al., 1991). Entretanto, um dos métodos mais utilizados, em levantamentos populacionais de insetos é o uso de armadilhas luminosas que são instrumentos destinados à captura de insetos fototrópicos positivos, ou seja, aqueles que habitualmente voam ao crepúsculo ou à noite (SILVEIRA NETO et al., 1976).

Especificamente no Rio Grande do Sul, estudos da entomofauna de Lepidoptera associada a cultivos de interesse econômico, com a utilização de armadilha luminosa, são pouco significativos, especialmente em cultivos florestais (TARRAGÓ et al., 1975; VIANA E COSTA, 2001). Cabe ressaltar, que os levantamentos entomofaunísticos são necessários à identificação das espécies com potencial de dano à cultura, bem como servirem de banco de dados para analisar a sustentabilidade ambiental dos florestamentos durante os anos de cultivo, pois os insetos podem constituir importantes bioindicadores de qualidade ambiental. Isso é reforçado por Lindgren e Borden (1983), os quais salientam que o conhecimento da composição da fauna de insetos em plantações florestais é um dos componentes básicos de um programa de manejo integrado de pragas.

Neste sentido, objetivou-se coletar, identificar e caracterizar faunisticamente a entomofauna de Lepidoptera associada a um plantio de Eucalyptus spp., no município de Pinheiro Machado, RS.

\section{MATERIAL E MÉTODOS}

As coletas foram realizadas no período de outubro de 2005 a outubro de 2007, em área florestada com Eucalyptus saligna (67,13 ha), Eucalyptus grandis (9,31 ha), Eucalyptus dunnii (4,77 ha) e diversos clones de Eucalyptus spp. (170,56 ha) com aproximadamente 2 anos de idade, pertencente à Empresa Votorantim Celulose \& Papel S/A, fazenda São Manoel, localizada às margens da rodovia BR 293 a $7 \mathrm{~km}$ da cidade de Pinheiro Machado, estado do Rio Grande do Sul (3133'51" S e 5325'59” W). A área onde se realizou o estudo ocupa 631,53 ha, dos quais 251,77 ha são florestados com espécies de eucalipto, sendo o restante, 379,76 ha ocupados por áreas de preservação permanente (mata nativa, banhado/brejo, campo nativo e afloramentos rochosos).

Para a coleta dos lepidópteros fototrópicos positivos foram utilizadas três armadilhas luminosas de modelo Al Intral (FT15T12BL). As armadilhas foram instaladas a $2 \mathrm{~m}$ do solo, distantes entre si por aproximadamente $300 \mathrm{~m}$, colocadas no interior do florestamento, providas de luz negra e acionadas quinzenalmente, durante uma noite, por bateria de 12 volts, no intervalo das $19 \mathrm{~h}$ às $7 \mathrm{~h}$. Na porção inferior da armadilha, foi fixado um saco plástico com capacidade de 50 litros, contendo no seu interior tiras de papel e no fundo um frasco com algodão embebido em acetato de etila. $\mathrm{O}$ objetivo

Ci. F1., v. 21, n. 4, out.-dez., 2011 
das tiras de papel, juntamente com o acetato, foi diminuir os danos morfológicos aos exemplares capturados (FERREIRA e MARTINS, 1982).

Após este período os insetos capturados foram transportados ao Laboratório de Biologia de Insetos (LBI), Departamento de Fitossanidade (DFs), Faculdade de Agronomia Eliseu Maciel (FAEM), Universidade Federal de Pelotas (UFPel), onde foram armazenados em freezer para posterior triagem, transfixação e identificação. A identificação foi realizada pelo taxonomista Dr. Eduardo José Ely e Silva DFs/FAEM/UFPel e pelo especialista Dr. Sinval Silveira Neto, Escola Superior de Agricultura "Luiz de Queiroz" (ESALQ/USP), mediante comparação com coleções entomológicas e uso de bibliografia especializada (SEITZ, 1944) .

Por meio do programa AnaFau (MORAES et al., 2003) foram calculados os índices faunísticos de frequência, abundância, dominância, constância e de diversidade de Shannon-Weaner (H').

\section{RESULTADOS E DISCUSSÃO}

Durante o período de estudo foram realizadas 48 coletas, sendo coletados 2.020 indivíduos, distribuídos em 14 famílias e 220 espécies. Das espécies coletadas, 160 foram identificadas em nível de gênero e/ou espécie e 60 identificadas somente por família, perfazendo 72,7 e $27,3 \%$ do total de indivíduos coletados, respectivamente. Dos fatores ambientais temperatura e precipitação, constatou-se que a temperatura afetou diretamente a flutuação populacional de lepidópteros, indicando que esses insetos podem aumentar suas populações em condições ambientais favoráveis e diminuir nas desfavoráveis, tendo essas condições influência na emergência dos insetos. Também convém mencionar que a maioria das espécies coletadas possui vários ciclos de desenvolvimento no decorrer de um ano, no entanto, parece haver uma sincronização na emergência dos adultos em períodos pré-definidos, especialmente após um período de temperatura elevada que incluem os meses de primavera e verão (setembro, outubro, novembro e dezembro) e nos meses que antecedem o inverno (março, abril e maio), (Figura 1).

As famílias Noctuidae, Arctiidae e Geometridae com 1.196, 385 e 212 exemplares, respectivamente, apresentaram o maior número de indivíduos coletados, perfazendo $88,77 \%$

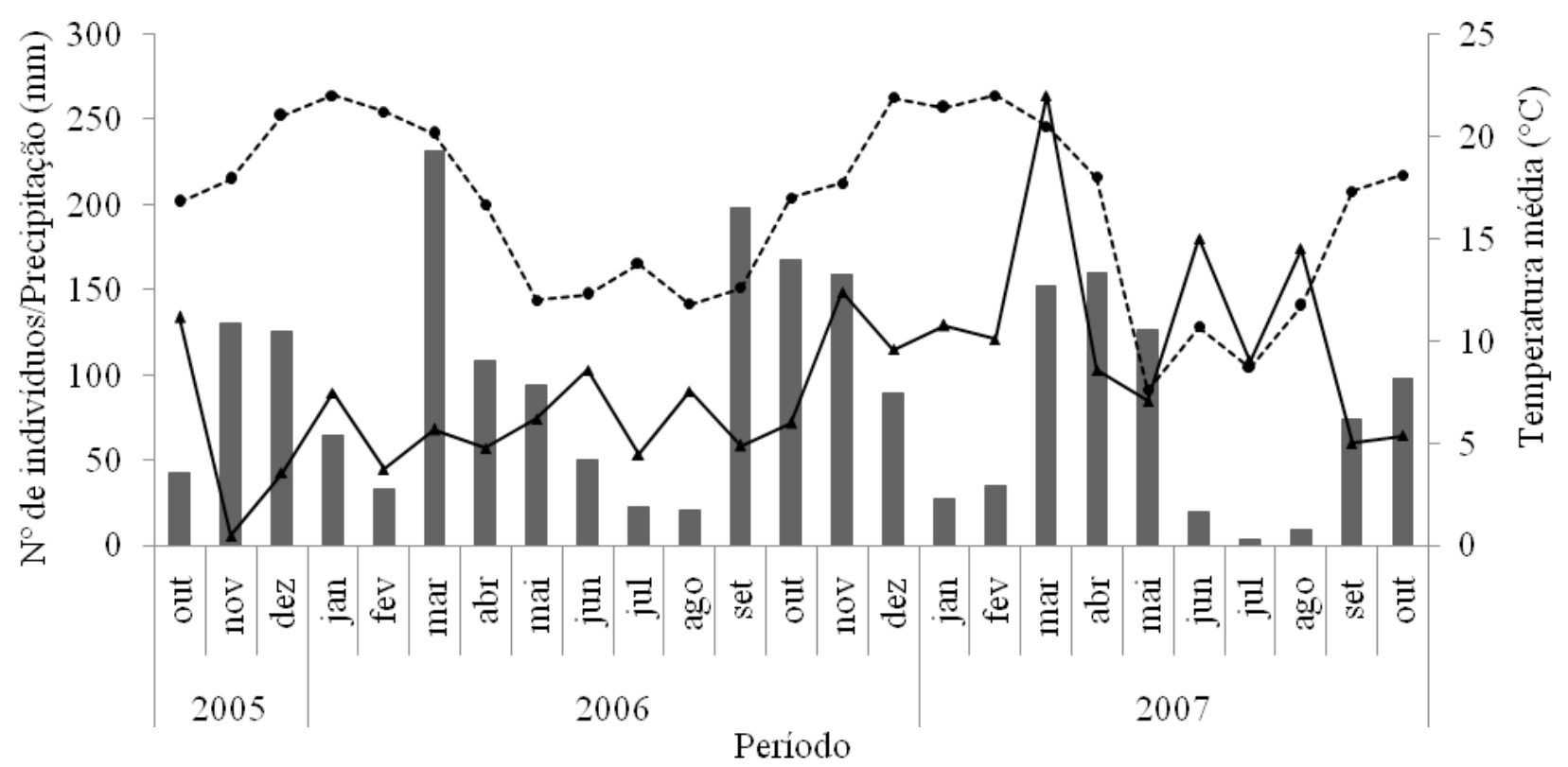

Número de indivíduos

—-Precipitação $(\mathrm{mm}) \quad$------ Temperatura média $\left({ }^{\circ} \mathrm{C}\right)$

FIGURA 1: Flutuação populacional de lepidópteros coletados com armadilhas luminosas em um plantio de Eucalyptus spp., no período de outubro de 2005 a outubro de 2007, no município de Pinheiro Machado, RS.

FIGURE 1: Population fluctuation of lepidopterous collected with light traps in a plantation of Eucalyptus spp., in the period of October 2005 to October 2007, in the Municipality of Pinheiro Machado, RS. 
do total de insetos (Tabela 1). A predominância de determinadas famílias pode estar associada à disponibilidade de alimento que favorece $\mathrm{o}$ estabelecimento e desenvolvimento dos insetos na área em estudo, bem como, essas podem ter sido mais atraídas pelas armadilhas luminosas do que outras espécies de Lepidoptera. Entretanto, frente à grande quantidade e diversidade de lepidópteros coletados convém ressaltar que as armadilhas luminosas são excelentes ferramentas para coleta e monitoramento de espécies de Lepidoptera em plantios florestais.

Em algumas famílias constatou-se a ocorrência de espécies desfolhadoras da cultura do eucalipto (Tabela 2). Em Saturniidae foram coletadas as espécies Automeris illustris e Eacles imperialis magnifica. Automeris illustris

TABELA 1: Número de indivíduos por família de Lepidoptera coletados com armadilhas luminosas em plantio de Eucalyptus spp., no período de outubro de 2005 a outubro de 2007, no município de Pinheiro Machado, RS.

TABLE 1: Number of individuals for Lepidoptera family collected with light traps in plantation of Eucalyptus spp., in the period of October 2005 to October 2007, in the Municipality of Pinheiro Machado, RS.

\begin{tabular}{lccccc}
\hline \multirow{2}{*}{ Família } & \multicolumn{5}{c}{ Número de insetos } \\
\cline { 2 - 6 } & 2005 & 2006 & 2007 & Total & $\%$ \\
\hline Aididae & - & - & 1 & 1 & 0,05 \\
Apatelodidae & 1 & 10 & 1 & 12 & 0,59 \\
Arctiidae & 38 & 268 & 79 & 385 & 19,06 \\
Cossidae & - & 2 & - & 2 & 0,10 \\
Geometridae & 3 & 119 & 90 & 212 & 10,50 \\
Lasiocampidae & 3 & 5 & 3 & 11 & 0,54 \\
Limacodidae & - & 5 & 22 & 27 & 1,34 \\
Lymatriidae & - & 1 & 3 & 4 & 0,20 \\
Megalopygidae & 1 & 1 & - & 2 & 0,10 \\
Mimallonidae & 1 & - & 1 & 2 & 0,10 \\
Noctuidae & 36 & 818 & 342 & 1196 & 59,21 \\
Notodontidae & - & 6 & 30 & 36 & 1,78 \\
Saturniidae & 1 & 50 & 23 & 74 & 3,66 \\
Sphingidae & 9 & 38 & 9 & 56 & 2,77 \\
\hline Total & 92 & 1324 & 604 & 2020 & 100,00 \\
\hline
\end{tabular}

foi encontrada causando desfolha de eucalipto em Minas Gerais, sendo considerada praga do eucalipto no Rio Grande do Sul (ZANUNCIO et al., 1991). Eacles imperialis magnifica ocorreu em surto no município de Linhares-ES, causando desfolha em cerca de 100 ha de Eucalyptus grandis (ZANUNCIO et al., 1993).

Dentre os Geometridae ocorreu a espécie Oxydia agliata que foi relatada desfolhando uma área de 250 ha de Eucalyptus cloeziana, em Alagoinhas-BA (SANTOS et al., 1993). No entanto, Sarcina violacens (Lymantriidae) é aquela que merece maior atenção pelo elevado potencial de dano em virtude de sua voracidade. No Brasil, Silva (1949) refere-se a este inseto em ataque a 50.000 árvores de Eucalyptus tereticornis, em Teresópolis-RJ.

As pragas da eucaliptocultura coletadas, mesmo sendo consideradas espécies raras, necessitam ser monitoradas, pois essas podem estar ocorrendo em baixo número devido à limitação de condições ambientais favoráveis ou pela competição com as espécies predominantes. No entanto, com as modificações ambientais decorrentes da expansão da eucaliptocultura na região esses insetos, futuramente, podem causar danos a esse ecossistema. Segundo Schowalter et al. (1986), isso pode ocorrer porque a estrutura da vegetação original determina a distribuição espacial e a disponibilidade de recursos para os herbívoros. Em função disso, as espécies que utilizam o eucalipto como alimento, nesse caso principalmente as espécies pragas, podem ter seu desenvolvimento favorecido e aumentar suas populações, fato que configura uma alteração no equilíbrio populacional anteriormente existente entre as diferentes espécies de insetos que habitavam aquele ambiente.

Diante disso, existe a necessidade de monitoramento da população das espécies pragas do eucalipto na região. Isso pode ser realizado com o uso de armadilhas luminosas, especialmente nos períodos do ano de temperatura mais elevada, mais especificamente no período de setembro a abril, quando foram verificados os picos populacionais. O monitoramento se faz necessário, pois estudos de levantamento populacional demonstram apenas a abundância das diferentes espécies em determinado ambiente ou ecossistema e geram poucas informações a respeito do ciclo, sazonalidade e dinâmica populacional ao longo do tempo (MORALES et al., 1999). 
TABELA 2: Análise faunística de lepidópteros coletados com armadilhas luminosas em plantio de Eucalyptus spp., no período de outubro de 2005 a outubro de 2007, no município de Pinheiro Machado, RS.

TABLE 2: Faunistic analysis of lepidopterous collected with ligth traps in a plantation of Eucalyptus spp., in the period of October 2005 to October 2007, in the Municipality of Pinheiro Machado, RS.

\begin{tabular}{|c|c|c|c|c|c|c|}
\hline Famílias/Espécies & Indiv. & $\%$ & $\mathrm{D}^{1}$ & A & $\mathrm{F}$ & $\mathrm{C}$ \\
\hline \multicolumn{7}{|l|}{ Aididae } \\
\hline Xernarchus osorius Herrich-Schäffer, 1816 & 1 & 0,06 & nd & $\mathrm{r}$ & $\mathrm{pf}$ & $\mathrm{z}$ \\
\hline \multicolumn{7}{|l|}{ Apatelodidae } \\
\hline Ephoria marginalis (Walker, 1856) & 2 & 0,11 & nd & $\mathrm{r}$ & $\mathrm{pf}$ & $\mathrm{z}$ \\
\hline Apatelodaes corema Schaus, 1897 & 1 & 0,06 & nd & $\mathrm{r}$ & pf & $\mathrm{z}$ \\
\hline Apatelodes sp. & 4 & 0,22 & nd & $\mathrm{r}$ & $\mathrm{pf}$ & $\mathrm{y}$ \\
\hline Apatelodes zikani Draudt, 1929 & 4 & 0,22 & nd & $\mathrm{r}$ & pf & $\mathrm{z}$ \\
\hline Olceclostera truncata (Walter, 1855) & 1 & 0,06 & nd & $\mathrm{r}$ & $\mathrm{pf}$ & $\mathrm{z}$ \\
\hline \multicolumn{7}{|l|}{ Arctiidae } \\
\hline Agoraea semivitrea Rotschild, 1909 & 1 & 0,06 & nd & $\mathrm{r}$ & $\mathrm{pf}$ & $\mathrm{z}$ \\
\hline Agylla argentifera (Walker, 1866) & 56 & 3,10 & $\mathrm{~d}$ & $\mathrm{ma}$ & $\mathrm{mf}$ & $\mathrm{w}$ \\
\hline Biturix rectilinea (Burmeister, 1878) & 34 & 1,88 & $\mathrm{~d}$ & $\mathrm{ma}$ & $\mathrm{mf}$ & $\mathrm{w}$ \\
\hline Dysschema centenaria (Burmeister, 1878) & 1 & 0,06 & nd & $\mathrm{r}$ & $\mathrm{pf}$ & $\mathrm{z}$ \\
\hline Dysschema hilarina (Weyner, 1914) & 4 & 0,22 & nd & $\mathrm{r}$ & pf & $\mathrm{y}$ \\
\hline Dysschema sacrigisa (Hübner, 1831) & 1 & 0,06 & nd & $\mathrm{r}$ & $\mathrm{pf}$ & $\mathrm{z}$ \\
\hline Eurota helena (Herrich-Schäffer, 1854) & 5 & 0,28 & nd & $\mathrm{r}$ & pf & $\mathrm{w}$ \\
\hline Eurota herricki Butter, 1876 & 11 & 0,61 & $\mathrm{~d}$ & $\mathrm{c}$ & $\mathrm{f}$ & $\mathrm{w}$ \\
\hline Hypercompe detecta (Obterthür, 1881) & 1 & 0,06 & nd & $\mathrm{r}$ & $\mathrm{pf}$ & $\mathrm{z}$ \\
\hline Hypercompe sp. (Walker, 1855) & 2 & 0,11 & nd & $\mathrm{r}$ & $\mathrm{pf}$ & $\mathrm{z}$ \\
\hline Hypercompe kinkelini (Burmeister, 1880) & 3 & 0,17 & nd & $\mathrm{r}$ & pf & $\mathrm{z}$ \\
\hline Hypidalia enervis (Schaus, 1894) & 2 & 0,11 & nd & $\mathrm{r}$ & $\mathrm{pf}$ & $\mathrm{z}$ \\
\hline Idalus agastus Dyar, 1910 & 6 & 0,33 & $\mathrm{~d}$ & $d$ & $\mathrm{pf}$ & $\mathrm{z}$ \\
\hline Leucanopsis leucanina (Felder \& Rogenhofer, 1874) & 14 & 0,77 & $\mathrm{~d}$ & a & $\mathrm{mf}$ & $\mathrm{y}$ \\
\hline Lophocampa dinora (Schaus, 1855) & 3 & 0,17 & nd & $\mathrm{r}$ & $\mathrm{pf}$ & $\mathrm{z}$ \\
\hline Lophocampa texta (Herrich-Shäffer, 1855) & 3 & 0,17 & nd & $\mathrm{r}$ & $\mathrm{pf}$ & $\mathrm{y}$ \\
\hline Melesa castrina Schaus, 1905 & 7 & 0,39 & $\mathrm{~d}$ & $\mathrm{c}$ & $\mathrm{f}$ & $\mathrm{w}$ \\
\hline Opharus rema (Dognin, 1891) & 3 & 0,17 & nd & $\mathrm{r}$ & $\mathrm{pf}$ & $\mathrm{z}$ \\
\hline Paracles azollae (Berg, 1877) & 26 & 1,44 & $\mathrm{~d}$ & ma & $\mathrm{mf}$ & $\mathrm{y}$ \\
\hline Paracles cajetani (Rothschild, 1910) & 128 & 7,08 & $\mathrm{~d}$ & $\mathrm{ma}$ & $\mathrm{mf}$ & $\mathrm{w}$ \\
\hline Paracles fusca (Walker, 1856) & 22 & 1,22 & $\mathrm{~d}$ & $\mathrm{ma}$ & $\mathrm{mf}$ & $\mathrm{w}$ \\
\hline Paracles uniformis (Jones, 1912) & 4 & 0,22 & nd & $\mathrm{r}$ & $\mathrm{pf}$ & $\mathrm{w}$ \\
\hline Paracles paula (Schaus, 1896) & 2 & 0,11 & nd & $\mathrm{r}$ & $\mathrm{pf}$ & $\mathrm{z}$ \\
\hline Paracles variegata (Schaus, 1904) & 3 & 0,17 & nd & $\mathrm{r}$ & $\mathrm{pf}$ & $\mathrm{z}$ \\
\hline Pelochyta cinerea (Walker,1855) & 4 & 0,22 & nd & $\mathrm{r}$ & pf & $\mathrm{w}$ \\
\hline Philoros affinis (Rothschild, 1912) & 39 & 2,16 & $\mathrm{~d}$ & $\mathrm{ma}$ & $\mathrm{mf}$ & $\mathrm{w}$ \\
\hline \multicolumn{7}{|l|}{ Cossidae } \\
\hline Givira sp. & 2 & 0,11 & nd & $\mathrm{r}$ & $\mathrm{pf}$ & $\mathrm{z}$ \\
\hline \multicolumn{7}{|l|}{ Geometridae } \\
\hline Acrotomodes sp. 1 & 4 & 0,22 & nd & $\mathrm{r}$ & $\mathrm{pf}$ & $\mathrm{z}$ \\
\hline Acrotomodes sp. 2 & 2 & 0,11 & nd & $\mathrm{r}$ & $\mathrm{pf}$ & $\mathrm{z}$ \\
\hline Acrotomodes sporadata Warren, 1905 & 1 & 0,06 & nd & $\mathrm{r}$ & $\mathrm{pf}$ & $\mathrm{z}$ \\
\hline Bryoptera sp. & 2 & 0,11 & nd & $\mathrm{r}$ & $\mathrm{pf}$ & $\mathrm{z}$ \\
\hline Epimecis marcida (Warren, 1906) & 1 & 0,06 & nd & $\mathrm{r}$ & pf & $\mathrm{z}$ \\
\hline Hymenomima amberia (Schaus, 1901) & 5 & 0,28 & nd & $\mathrm{r}$ & pf & $\mathrm{z}$ \\
\hline
\end{tabular}


TABELA 2: Continuação ...

TABLE 2: Continued ...

\begin{tabular}{|c|c|c|c|c|c|c|}
\hline Famílias/Espécies & Indiv. & $\%$ & $\mathrm{D}^{1}$ & $\mathrm{~A}$ & $\mathrm{~F}$ & $\mathrm{C}$ \\
\hline Iridopis lurida (Schaus, 1918) & 1 & 0,06 & nd & $\mathrm{r}$ & $\mathrm{pf}$ & $\mathrm{z}$ \\
\hline Lissochlora nortia (Druce, 1892) & 2 & 0,11 & nd & $\mathrm{r}$ & $\mathrm{pf}$ & $\mathrm{z}$ \\
\hline Lomographa nubimargo Warren, 1897 & 2 & 0,11 & nd & $\mathrm{r}$ & $\mathrm{pf}$ & $\mathrm{z}$ \\
\hline Macaria regulata (Fabricius, 1775) & 2 & 0,11 & nd & $\mathrm{r}$ & $\mathrm{pf}$ & $\mathrm{z}$ \\
\hline Microsema gladiaria (Guenée, 1858) & 10 & 0,55 & $\mathrm{~d}$ & $\mathrm{c}$ & $\mathrm{f}$ & $\mathrm{w}$ \\
\hline Microsema subcarnea (Warren, 1895) & 3 & 0,17 & nd & $\mathrm{r}$ & $\mathrm{pf}$ & $\mathrm{z}$ \\
\hline Oospila pallidaria Schaus, 1898 & 2 & 0,11 & nd & $\mathrm{r}$ & $\mathrm{pf}$ & $\mathrm{z}$ \\
\hline Oxydia agliata Guenée, 1858 & 3 & 0,17 & nd & $\mathrm{r}$ & $\mathrm{pf}$ & $\mathrm{z}$ \\
\hline Oxydia chalybeata Warren, 1897 & 1 & 0,06 & nd & $\mathrm{r}$ & $\mathrm{pf}$ & $\mathrm{z}$ \\
\hline Oxydia peosinata Guenée, 1858 & 8 & 0,44 & $\mathrm{~d}$ & $\mathrm{c}$ & $\mathrm{f}$ & $\mathrm{z}$ \\
\hline Pero gonopteraria (Guenée, 1858) & 9 & 0,50 & $\mathrm{~d}$ & $\mathrm{c}$ & $\mathrm{f}$ & $\mathrm{w}$ \\
\hline Pero hoedularia (Guenée, 1858) & 1 & 0,06 & nd & $\mathrm{r}$ & $\mathrm{pf}$ & $\mathrm{z}$ \\
\hline Pero refellaria (Guenée, 1858) & 3 & 0,17 & nd & $\mathrm{r}$ & $\mathrm{pf}$ & $\mathrm{z}$ \\
\hline Pero sp. 1 & 3 & 0,17 & nd & $\mathrm{r}$ & $\mathrm{pf}$ & $\mathrm{z}$ \\
\hline Pero sp. 2 & 1 & 0,06 & nd & $\mathrm{r}$ & $\mathrm{pf}$ & $\mathrm{z}$ \\
\hline Pero sp. 3 & 1 & 0,06 & nd & $\mathrm{r}$ & $\mathrm{pf}$ & $\mathrm{z}$ \\
\hline Pherotesia condensaria (Guenée, 1858) & 1 & 0,06 & nd & $\mathrm{r}$ & $\mathrm{pf}$ & $\mathrm{z}$ \\
\hline Phrygionis modesta Warren, 1904 & 4 & 0,22 & nd & $\mathrm{r}$ & $\mathrm{pf}$ & $\mathrm{y}$ \\
\hline Physocleora fusca Warren, 1897 & 2 & 0,11 & nd & $\mathrm{r}$ & $\mathrm{pf}$ & $\mathrm{z}$ \\
\hline Physocleora philaria (Guenée, 1858) & 1 & 0,06 & nd & $\mathrm{r}$ & $\mathrm{pf}$ & $\mathrm{z}$ \\
\hline Prochoerodes tetragonata Guenée, 1858 & 3 & 0,17 & nd & $\mathrm{r}$ & $\mathrm{pf}$ & $\mathrm{z}$ \\
\hline Sabulodes exhonorata Guenée, 1858 & 1 & 0,06 & nd & $\mathrm{r}$ & $\mathrm{pf}$ & $\mathrm{z}$ \\
\hline Synchlora frondaria Guenée, 1857 & 4 & 0,22 & nd & $\mathrm{r}$ & $\mathrm{pf}$ & $\mathrm{z}$ \\
\hline Tachypyle oliva (Schaus, 1901) & 4 & 0,22 & nd & $\mathrm{r}$ & $\mathrm{pf}$ & $\mathrm{y}$ \\
\hline \multicolumn{7}{|l|}{ Lasiocampidae } \\
\hline Artace cribaria (Ljungh, 1825) & 2 & 0,11 & nd & $\mathrm{r}$ & $\mathrm{pf}$ & $\mathrm{z}$ \\
\hline Euglyphis deusta (Herrich-Schäffer, 1854) & 1 & 0,06 & nd & $\mathrm{r}$ & $\mathrm{pf}$ & $\mathrm{z}$ \\
\hline Euglyphis lacrimosa Schaus, 1892 & 3 & 0,17 & nd & $\mathrm{r}$ & $\mathrm{pf}$ & $\mathrm{z}$ \\
\hline Euglyphis lignosa (Walker,1855) & 1 & 0,06 & nd & $\mathrm{r}$ & $\mathrm{pf}$ & $\mathrm{z}$ \\
\hline Euglyphis sp. & 3 & 0,17 & nd & $\mathrm{r}$ & $\mathrm{pf}$ & $\mathrm{z}$ \\
\hline Tolype ventriosa Draudt, 1927 & 1 & 0,06 & nd & $\mathrm{r}$ & $\mathrm{pf}$ & $\mathrm{z}$ \\
\hline \multicolumn{7}{|l|}{ Limacodidae } \\
\hline Perola petropolis Dyar, 1905 & 3 & 0,17 & nd & $\mathrm{r}$ & $\mathrm{pf}$ & $\mathrm{z}$ \\
\hline Perola chica Jones, 1912 & 1 & 0,06 & nd & $\mathrm{r}$ & $\mathrm{pf}$ & $\mathrm{z}$ \\
\hline \multicolumn{7}{|l|}{ Lymantriidae } \\
\hline Sarcina violascens (Herrich-Schäffer, 1856) & 2 & 0,11 & nd & $\mathrm{r}$ & $\mathrm{pf}$ & $\mathrm{z}$ \\
\hline \multicolumn{7}{|l|}{ Megalopygidae } \\
\hline Norape albilineata Hopp. 1927 & 1 & 0,06 & nd & $\mathrm{r}$ & $\mathrm{pf}$ & $\mathrm{z}$ \\
\hline Podalia walkeri (Berg, 1882) & 4 & 0,22 & nd & $\mathrm{r}$ & $\mathrm{pf}$ & $\mathrm{z}$ \\
\hline \multicolumn{7}{|l|}{ Mimallonidae } \\
\hline Vanenga mera (Dognin, 1924) & 1 & 0,06 & nd & $\mathrm{r}$ & $\mathrm{pf}$ & $\mathrm{z}$ \\
\hline \multicolumn{7}{|l|}{ Noctuidae } \\
\hline Achaea ablunaris (Guenée, 1852) & 1 & 0,06 & nd & $\mathrm{r}$ & $\mathrm{pf}$ & $\mathrm{z}$ \\
\hline Acontia ardoris Hübner, 1831 & 2 & 0,11 & nd & $\mathrm{r}$ & $\mathrm{pf}$ & $\mathrm{z}$ \\
\hline Acontia morides Schaus, 1894 & 5 & 0,28 & nd & $\mathrm{r}$ & $\mathrm{pf}$ & $\mathrm{w}$ \\
\hline Agrotis bachystria (Hampson, 1903) & 3 & 0,17 & nd & $\mathrm{r}$ & $\mathrm{pf}$ & $\mathrm{y}$ \\
\hline Agrotis bosqui (Khöler, 1945) & 3 & 0,17 & nd & $\mathrm{r}$ & $\mathrm{pf}$ & $\mathrm{z}$ \\
\hline Agrotis gypaetina Guenée, 1852 & 13 & 0,72 & $\mathrm{~d}$ & $\mathrm{c}$ & $\mathrm{f}$ & $\mathrm{w}$ \\
\hline
\end{tabular}


TABELA 2: Continuação ...

TABLE 2: Continued ...

\begin{tabular}{|c|c|c|c|c|c|c|}
\hline Famílias/Espécies & Indiv. & $\%$ & $\mathrm{D}^{1}$ & $\mathrm{~A}$ & $\mathrm{~F}$ & $\mathrm{C}$ \\
\hline Agrotis ipsilon (Hufnagel, 1766) & 1 & 0,06 & nd & $\mathrm{r}$ & $\mathrm{pf}$ & $\mathrm{Z}$ \\
\hline Agrotis malefida Guenée, 1852 & 56 & 3,10 & $d$ & $\mathrm{ma}$ & $\mathrm{mf}$ & $\mathrm{W}$ \\
\hline Agrotis subterranea (Fabricius, 1794) & 32 & 1,77 & $\mathrm{~d}$ & $\mathrm{ma}$ & $\mathrm{mf}$ & $\mathrm{W}$ \\
\hline Amphipyra problematica Draudt, 1924 & 1 & 0,06 & nd & $\mathrm{r}$ & $\mathrm{pf}$ & $\mathrm{Z}$ \\
\hline Anicla ignicans (Guenée, 1852) & 1 & 0,06 & nd & $\mathrm{r}$ & $\mathrm{pf}$ & $\mathrm{Z}$ \\
\hline Anicla infecta (Ochsenheimer, 1816) & 19 & 1,05 & $d$ & $\mathrm{ma}$ & $\mathrm{mf}$ & $\mathrm{W}$ \\
\hline Anomis erosa Hübner, 1816 & 1 & 0,06 & nd & $\mathrm{r}$ & $\mathrm{pf}$ & $\mathrm{Z}$ \\
\hline Anticarsia gemmatalis Hübner, 1818 & 3 & 0,17 & nd & $\mathrm{r}$ & $\mathrm{pf}$ & $\mathrm{y}$ \\
\hline Ascalapha odorata (Linnaeus, 1758) & 2 & 0,11 & nd & $\mathrm{r}$ & $\mathrm{pf}$ & $\mathrm{Z}$ \\
\hline Aucula hilzingeri (Berg, 1882) & 8 & 0,44 & $\mathrm{~d}$ & $\mathrm{c}$ & $\mathrm{f}$ & $\mathrm{W}$ \\
\hline Autographa bomaeriensis (Berg, 1882) & 1 & 0,06 & nd & $\mathrm{r}$ & $\mathrm{pf}$ & $\mathrm{Z}$ \\
\hline Basigara repanda (Fabricius, 1793) & 2 & 0,11 & nd & $\mathrm{r}$ & $\mathrm{pf}$ & $\mathrm{Z}$ \\
\hline Bryolymnia bicon (Druce, 1889) & 9 & 0,50 & $\mathrm{~d}$ & $\mathrm{c}$ & f & $\mathrm{W}$ \\
\hline Chabuata major (Guenée, 1852) & 17 & 0,94 & $\mathrm{~d}$ & $\mathrm{ma}$ & $\mathrm{mf}$ & $\mathrm{W}$ \\
\hline Chabuata rhodomelania (Kholer, 1959) & 1 & 0,06 & nd & $\mathrm{r}$ & $\mathrm{pf}$ & $\mathrm{Z}$ \\
\hline Condica sutor (Gueneé, 1852) & 1 & 0,06 & nd & $\mathrm{r}$ & $\mathrm{pf}$ & $\mathrm{Z}$ \\
\hline Condica vacilans (Walker, 1858) & 3 & 0,17 & nd & $\mathrm{r}$ & $\mathrm{pf}$ & $\mathrm{y}$ \\
\hline Cucullia argyrina (Guenée, 1852) & 1 & 0,06 & nd & $\mathrm{r}$ & $\mathrm{pf}$ & $\mathrm{Z}$ \\
\hline Cucullia heinrichi Köhler, 1952 & 2 & 0,11 & nd & $\mathrm{r}$ & $\mathrm{pf}$ & $\mathrm{Z}$ \\
\hline Dargida meridionalis (Hampson, 1905) & 2 & 0,11 & nd & $\mathrm{r}$ & $\mathrm{pf}$ & $\mathrm{Z}$ \\
\hline Elaphria mucicolora (Gueneé, 1852) & 22 & 1,22 & $\mathrm{~d}$ & $\mathrm{ma}$ & $\mathrm{mf}$ & $\mathrm{W}$ \\
\hline Elaphria villicosta (Walker, 1858) & 17 & 0,94 & $\mathrm{~d}$ & $\mathrm{ma}$ & $\mathrm{mf}$ & $\mathrm{Z}$ \\
\hline Eriopyga approximans Jones, 1908 & 120 & 6,63 & $\mathrm{~d}$ & $\mathrm{ma}$ & $\mathrm{mf}$ & $\mathrm{W}$ \\
\hline Eriopyga niveipuncta (Schaus, 1894) & 2 & 0,11 & nd & $\mathrm{r}$ & $\mathrm{pf}$ & $\mathrm{Z}$ \\
\hline Faronta albilinea (Hübner, 1821) & 23 & 1,27 & $\mathrm{~d}$ & $\mathrm{ma}$ & $\mathrm{mf}$ & $\mathrm{W}$ \\
\hline Helicoverpa zea (Boddie, 1850) & 4 & 0,22 & nd & $\mathrm{r}$ & $\mathrm{pf}$ & $\mathrm{y}$ \\
\hline Heliothis virescens (Fabricius, 1777) & 13 & 0,72 & $\mathrm{~d}$ & $\mathrm{c}$ & $\mathrm{f}$ & $\mathrm{W}$ \\
\hline Herminodes carbonelli Biezanko, 1959 & 13 & 0,72 & $\mathrm{~d}$ & $\mathrm{c}$ & $\mathrm{f}$ & $\mathrm{W}$ \\
\hline Herminodes lignea Schaus, 1904 & 4 & 0,22 & nd & $\mathrm{r}$ & $\mathrm{pf}$ & $\mathrm{y}$ \\
\hline Lasiestra consectatrix Draudt, 1924 & 5 & 0,28 & nd & $\mathrm{r}$ & $\mathrm{pf}$ & $\mathrm{y}$ \\
\hline Leucania humidicola Guenée, 1852 & 58 & 3,21 & $\mathrm{~d}$ & $\mathrm{ma}$ & $\mathrm{mf}$ & $\mathrm{W}$ \\
\hline Leucania jaliscana Schaus, 1898 & 42 & 2,32 & $\mathrm{~d}$ & $\mathrm{ma}$ & $\mathrm{mf}$ & $\mathrm{W}$ \\
\hline Leucania microsthica (Hampson, 1905) & 10 & 0,55 & $\mathrm{~d}$ & $\mathrm{c}$ & $\mathrm{f}$ & $\mathrm{W}$ \\
\hline Leucania rivorum Guenée, 1852 & 12 & 0,66 & d & $\mathrm{c}$ & $\mathrm{f}$ & $\mathrm{y}$ \\
\hline Macapta lurida (Schaus, 1898) & 19 & 1,05 & $\mathrm{~d}$ & ma & $\mathrm{mf}$ & $\mathrm{W}$ \\
\hline Magusa orbifera (Walker, 1857) & 5 & 0,28 & nd & $\mathrm{r}$ & $\mathrm{pf}$ & $\mathrm{y}$ \\
\hline Melipotis fasciolaris (Hübner, 1831) & 1 & 0,06 & nd & $\mathrm{r}$ & $\mathrm{pf}$ & $\mathrm{Z}$ \\
\hline Melipotis perpendicularis (Guenée, 1852) & 2 & 0,11 & nd & $\mathrm{r}$ & $\mathrm{pf}$ & $\mathrm{Z}$ \\
\hline Mocis latipes (Guenée, 1852) & 4 & 0,22 & $\mathrm{~d}$ & $\mathrm{ma}$ & $\mathrm{mf}$ & $\mathrm{W}$ \\
\hline Ochropleura cirphisioides Köhler, 1955 & 241 & 13,32 & $\mathrm{~d}$ & $\mathrm{ma}$ & $\mathrm{mf}$ & $\mathrm{W}$ \\
\hline Ophisma tropicalis Guenée, 1852 & 2 & 0,11 & nd & $\mathrm{r}$ & $\mathrm{pf}$ & $\mathrm{Z}$ \\
\hline Orthodes infirma Guenée, 1852 & 4 & 0,22 & nd & $\mathrm{r}$ & $\mathrm{pf}$ & $\mathrm{y}$ \\
\hline Peridroma saucia (Hübner, 1808) & 5 & 0,28 & nd & $\mathrm{r}$ & $\mathrm{pf}$ & $\mathrm{W}$ \\
\hline Pseudaletia adultera (Schaus, 1894) & 2 & 0,11 & nd & $\mathrm{r}$ & $\mathrm{pf}$ & $\mathrm{Z}$ \\
\hline Pseudaletia sequax Franclemont, 1952 & 14 & 0,77 & $\mathrm{~d}$ & $\mathrm{c}$ & $\mathrm{f}$ & $\mathrm{W}$ \\
\hline Pseudoleucania tendiliense (Köhler, 1955) & 143 & 7,90 & $\mathrm{~d}$ & $\mathrm{ma}$ & $\mathrm{mf}$ & $\mathrm{W}$ \\
\hline Rachiplusia nu (Guenée, 1852) & 15 & 0,83 & $\mathrm{~d}$ & $\mathrm{c}$ & $\mathrm{f}$ & $\mathrm{W}$ \\
\hline Spodoptera cosmioides (Walker, 1858) & 57 & 3,15 & $\mathrm{~d}$ & $\mathrm{ma}$ & $\mathrm{mf}$ & $\mathrm{W}$ \\
\hline
\end{tabular}


TABELA 2: Continuação ...

TABLE 2: Continued ...

\begin{tabular}{|c|c|c|c|c|c|c|}
\hline Famílias/Espécies & Indiv. & $\%$ & $\mathrm{D}^{1}$ & $\mathrm{~A}$ & $\mathrm{~F}$ & $\mathrm{C}$ \\
\hline Spodoptera eridania (Stoll, 1782) & 7 & 0,39 & $\mathrm{~d}$ & $\mathrm{c}$ & $\mathrm{f}$ & $\mathrm{Z}$ \\
\hline Spodoptera frugiperda (J.E. Smith, 1797) & 6 & 0,33 & $d$ & $\mathrm{c}$ & $\mathrm{f}$ & $\mathrm{Z}$ \\
\hline Spodoptera marima (Schaus, 1904) & 7 & 0,39 & $\mathrm{~d}$ & $\mathrm{c}$ & $\mathrm{f}$ & $\mathrm{w}$ \\
\hline Tripseuxoa strigata Hampson, 1903 & 60 & 3,32 & $\mathrm{~d}$ & $\mathrm{ma}$ & $\mathrm{mf}$ & $\mathrm{w}$ \\
\hline Zale pachystrigata Hampson, 1913 & 4 & 0,22 & nd & $\mathrm{r}$ & $\mathrm{pf}$ & $\mathrm{y}$ \\
\hline \multicolumn{7}{|l|}{ Notodontidae } \\
\hline Eustema rapana Jones, 1908 & 27 & 1,49 & $\mathrm{~d}$ & $\mathrm{ma}$ & $\mathrm{mf}$ & $\mathrm{w}$ \\
\hline Hippia pronax Dognin, 1908 & 1 & 0,06 & nd & $\mathrm{r}$ & $\mathrm{pf}$ & $\mathrm{Z}$ \\
\hline Marthula castrensis Schaus, 1906 & 4 & 0,22 & nd & $\mathrm{r}$ & $\mathrm{pf}$ & $\mathrm{y}$ \\
\hline Misogada bleura Schaus, 1915 & 1 & 0,06 & nd & $\mathrm{r}$ & $\mathrm{pf}$ & $\mathrm{Z}$ \\
\hline Rhuda labella Dyar, 1908 & 1 & 0,06 & nd & $\mathrm{r}$ & $\mathrm{pf}$ & $\mathrm{Z}$ \\
\hline Tecmessa annulipes Berg, 1878 & 2 & 0,11 & nd & $\mathrm{r}$ & $\mathrm{pf}$ & $\mathrm{Z}$ \\
\hline \multicolumn{7}{|l|}{ Saturniidae } \\
\hline Arsenura biundulata Schaus, 1906 & 11 & 0,61 & d & $\mathrm{c}$ & $\mathrm{f}$ & $\mathrm{Z}$ \\
\hline Automeris illustris (Walker, 1855) & 6 & 0,33 & $\mathrm{~d}$ & $\mathrm{r}$ & $\mathrm{pf}$ & $\mathrm{Z}$ \\
\hline Automeris naranja Schaus, 1898 & 1 & 0,06 & nd & $\mathrm{r}$ & $\mathrm{pf}$ & $\mathrm{Z}$ \\
\hline Citheronia brissoti (Boisduval, 1868) & 5 & 0,28 & nd & $\mathrm{r}$ & $\mathrm{pf}$ & $\mathrm{w}$ \\
\hline Coloradia sp. & 3 & 0,17 & nd & $\mathrm{r}$ & $\mathrm{pf}$ & $\mathrm{Z}$ \\
\hline Dirphia baroma (Schaus, 1906) & 5 & 0,28 & nd & $\mathrm{r}$ & $\mathrm{pf}$ & $\mathrm{W}$ \\
\hline Eacles imperialis magnifica Walker, 1855 & 1 & 0,06 & nd & $\mathrm{r}$ & $\mathrm{pf}$ & $\mathrm{Z}$ \\
\hline Eudyaria zeta (Berg, 1885) & 18 & 1,00 & $\mathrm{~d}$ & $\mathrm{ma}$ & $\mathrm{mf}$ & $\mathrm{y}$ \\
\hline Hyperchiria incisa Walker, 1855 & 2 & 0,11 & nd & $\mathrm{r}$ & $\mathrm{pf}$ & $\mathrm{Z}$ \\
\hline Lonomia obliqua Walker, 1855 & 3 & 0,17 & nd & $\mathrm{r}$ & $\mathrm{pf}$ & $\mathrm{Z}$ \\
\hline Lonomia sp. & 3 & 0,17 & nd & $\mathrm{r}$ & $\mathrm{pf}$ & $\mathrm{Z}$ \\
\hline Otherene purpurascens (Schaus, 1905) & 2 & 0,11 & nd & $\mathrm{r}$ & $\mathrm{pf}$ & $\mathrm{Z}$ \\
\hline Periga circunstans Walker, 1855 & 14 & 0,77 & $\mathrm{~d}$ & $\mathrm{c}$ & $\mathrm{f}$ & $\mathrm{Z}$ \\
\hline \multicolumn{7}{|l|}{ Sphingidae } \\
\hline Adhemarius gannascus (Stoll, 1790) & 5 & 0,28 & nd & $\mathrm{r}$ & $\mathrm{pf}$ & $\mathrm{w}$ \\
\hline Eumorpha sp. & 1 & 0,06 & nd & $\mathrm{r}$ & $\mathrm{pf}$ & $\mathrm{Z}$ \\
\hline Eumorpha vitis (Linnaeus, 1758) & 1 & 0,06 & nd & $\mathrm{r}$ & $\mathrm{pf}$ & $\mathrm{Z}$ \\
\hline Manduca armantipes (Rothschid \& Jordan, 1916) & 1 & 0,06 & nd & $\mathrm{r}$ & $\mathrm{pf}$ & $\mathrm{Z}$ \\
\hline Manduca florestan (Stoll, 1782) & 8 & 0,44 & $\mathrm{~d}$ & $\mathrm{c}$ & $\mathrm{f}$ & $\mathrm{Z}$ \\
\hline Manduca sexta paphus (Cramer, 1779) & 31 & 1,71 & $\mathrm{~d}$ & $\mathrm{ma}$ & $\mathrm{mf}$ & $\mathrm{w}$ \\
\hline Xylophanes tersa (Linnaeus, 1771) & 9 & 0,50 & $\mathrm{~d}$ & $\mathrm{c}$ & $\mathrm{f}$ & $\mathrm{w}$ \\
\hline Total & 1809 & 100,00 & - & - & - & - \\
\hline
\end{tabular}

Em que: $\mathrm{D}$ = Dominância - d: dominante; nd: não dominante; $\mathrm{A}=$ Abundância - ma: muito abundante; c: comum; d: dispersa; r: rara; $\mathrm{F}=$ Frequência - mf: muito frequente; f: frequente; pf: pouco frequente; $\mathrm{C}=$ Constância - w: constante; y: acessória; z: acidental; ${ }^{1}$ Dominância: Método de Laroca e Mielke.

De acordo com os dados contidos na Tabela 2 foram consideradas espécies predominantes por obterem os índices máximos de classificação faunística, como dominante, muito abundante, muito frequente e constante, as espécies Agylla argentifera, Biturix rectilinea, Paracles cajetani, Paracles fusca, Philoros affinis (Arctiidae), Agrotis malefida, Agrotis subterranea, Anicla infecta, Chabuata major, Elaphria mucicolora, Eriopyga approximans, Faronta albilinea, Leucania humidicola, Leucania jabiscana, Macapta lurida, Ochropleura cirphisioides, Pseudoleucania tendiliense, Spodoptera cosmioides, Tripseuxoa strigata (Noctuidae), Eustema rapana (Notodontidae) e Manduca sexta paphus (Sphingidae), perfazendo 68,89\% do total de insetos identificados por gênero e/ou espécie.

Quanto à dominância, verificou-se que das 160 espécies coletadas, 49 foram consideradas dominantes (Tabela 2). A maior quantidade de 
espécies não dominantes deve-se ao fato de que algumas ocorreram em número excessivo, o que contribuiu para que menos espécies sejam categorizadas como dominantes, pois o índice de dominância leva em consideração o número de insetos coletados, e sua categorização é feita através da frequência. De acordo com Silveira Neto et al. (1976), insetos dominantes têm a capacidade de modificar um impacto recebido do ambiente em benefício próprio, podendo ocasionar o aparecimento ou desaparecimento de outros organismos. Isso pode futuramente ocorrer nas áreas de cultivo de eucalipto da região, onde os insetos dominantes podem aumentar sua população devido, especialmente, à oferta de alimento, podendo explicar o surgimento de lepidópteros-praga, antes de pouca, ou nenhuma importância, à eucaliptocultura (BERNARDI et al., 2008).

Em relação à classificação de abundância, 74 espécies foram categorizadas como raras. Porém mesmo sendo consideradas raras, são muito importantes por apresentarem elevada influência sobre a diversidade dos ecossistemas. De acordo com Krebs (1972), as espécies raras, exprimem à alta diversidade de um sistema que evolui em ambiente de sucessão ecológica, tendo às comunidades a característica marcante de conter grande número de espécies raras, no entanto, esse número pode diminuir quando se despende maior esforço amostral.

Com relação à constância, 74 espécies foram categorizadas como acidentais. Tal fato relacionase a uma característica da área estudada, a qual possui muitas espécies com pequena quantidade de indivíduos, bem como esses não apresentam constância de coleta. Segundo Clemente (1995), uma alta percentagem de espécies acidentais, em comunidades florestais, indica uma resistência do meio à proliferação destas espécies, enquanto as espécies classificadas como muito abundantes, constantes e dominantes indicam o estabelecimento dessas espécies dentro das comunidades.

De acordo com Silveira Neto et al. (1995), após o conhecimento da fauna e do uso de análise faunística é possível se avaliar o impacto ambiental dos cultivos florestais por meio do estudo de espécies de insetos consideradas bioindicadoras de qualidade ambiental. Diante disso, as espécies predominantes, mesmo não apresentando grande importância econômica para a eucaliptocultura, são importantes indicadores de qualidade ambiental, por mostrarem a heterogeneidade da fauna de insetos na região e representarem fontes de alimento para inimigos naturais, especialmente parasitóides e predadores dos lepidópteros-praga do eucalipto.

A grande quantidade de espécies acidentais associadas ao elevado índice de diversidade $\left(H^{\prime}=3,891\right)$, mostram um ambiente equilibrado onde a competição interespecífica e intraespecífica podem determinar o comportamento das espécies. Cabe ressaltar que com a expansão dos florestamentos o índice de diversidade pode sofrer alterações. Acredita-se que os valores tendem a baixar, pois com a uniformização da vegetação, algumas espécies ou grupos restritos de insetos podem ser favorecidos e a grande maioria das espécies desfavorecidas. No entanto, caso a fragmentação dos cultivos com áreas de preservação permanente como mata nativa e campos sejam assegurados, o índice de diversidade pode tomar valores maiores dos verificados no presente estudo. Ainda convém mencionar que esse índice, em estudo similar, pode ser utilizado como comparativo para avaliação dos possíveis impactos da eucaliptocultura sobre a entomofauna de lepidópteros da região.

\section{CONCLUSÕES}

O florestamento de eucalipto estudado apresenta elevada diversidade de espécies de Lepidoptera.

A maioria dos lepidópteros identificados em nível de gênero e espécie, segundo a classificação faunística, são categorizados como não dominantes, pouco frequentes, raros e acidentais.

Os lepidópteros desfolhadores do eucalipto associados ao florestamento estudado são Oxydia agliata, Sarsina violascens, Automeris illustris, Eacles imperialis magnifica e Lonomia obliqua.

A ocorrência de lepidópteros-praga justifica a implementação de um programa de monitoramento desses insetos em florestamentos de eucalipto do Sul do Rio Grande do Sul. Adicionalmente, a coleta e monitoramento de lepidópteros-praga com armadilhas luminosas devem ser realizados nas épocas mais quentes do ano.

\section{REFERÊNCIAS BIBLIOGRÁFICAS}

BERNARDI, O. et al. Ocorrência de Euetheola humilis (Burmeister) (Coleoptera: Scarabaeidae) em Eucalyptus saligna Smith (Myrtaceae), no Estado do Rio Grande do Sul. Neotropical Entomology, Vacaria, v. 37, n. 1, jan./fev. 2008. 
BERTI FILHO, E. Insetos associados a plantações de espécies do gênero Eucalyptus nos Estados da Bahia, Espírito Santo, Mato Grosso do Sul, Minas Gerais e São Paulo. 1981. 176 p. Tese (Livre Docência em Entomologia) - Universidade de São Paulo. Escola Superior de Agricultura "Luiz de Queiroz", Piracicaba1981.

CLEMENTE, A. T. C. Análise de populações de Lepidoptera em comunidades florestais de Araucaria angustifolia, Eucalyptus grandis e Pinus taeda. 1995, 75 p. Dissertação. (Mestrado em Engenharia Florestal) - Universidade Federal do Paraná, Curitiba, 1995.

FERREIRA, P. S. F.; MARTINS, D. S. Contribuição ao método de captura de insetos por meio de armadilha luminosa, para a obtenção de exemplares sem danos morfológicos. Revista Ceres, Viçosa, v. 29, n. 165, p. 538-543, nov./dez. 1982.

KREBS, C. J. Ecology - The experimental analysis of distribution and abundance, 2 nd ed. Cambrigge: Harper and Row, 1972, 694 p.

LINDGREN, B. S.; BORDEN, J. H. Survey and mass trapping of ambrosia beetles (Coleoptera: Scolytidae) in timber processing areas on Vancouver Island. Canadian Journal of Forest Research, Ottawa, v. 13, n. 3, p. 481-493, June 1983.

MORAES, R. C. B.; HADDAD, M. L.; SILVEIRA NETO, S. et al. Software para análise faunística - AnaFau. In: SIMPÓSIO DE CONTROLE BIOLÓGICO, 2003, São Pedro. Resumos... São Pedro, 2003. p. 195.

MORALES, N. E. et al. Índices populacionais de besouros Scolytidae em reflorestamento de Eucalyptus grandis W. Hill ex. Maiden no município de Antônio Dias, Minas Gerais. Revista Árvore, Viçosa, v. 23, n. 3, p. 359-363, maio/jun. 1999.

RISCH, S. J.; ANDOW, D.; ALTIERI, M. A. Agroecosystem diversity and pest control: data, tentative conclusions, and new research directions. Environmental Entomology, Lanham, v. 12, n. 3, p. 625-629, June 1983.

SANTOS, G. P.; ZANUNCIO, J. C.; ANJOS, N. Novos resultados sobre a biologia de Psorocampa denticulata Schaus (Lepidoptera: Notodontidae), desfolhadora de eucalipto. Revista Árvore, Viçosa, v. 6, n. 2, p. 212-32, jun./jul. 1982.

SANTOS, G. P. et al. Descrição das lagartas desfolhadoras. In: ZANUNCIO, J. C. (Coord.). Manual de pragas em florestas. Lepidoptera desfolhadores de eucalipto: biologia, ecologia e controle. Viçosa: Folha de Viçosa, 1993, p. 12-66. SCHOWALTER, T. D.; HARGROVE, W. W.; CROSSLEY JR., D. A. Herbivory in forested ecosystems. Annual Review of Entomology, Palo Alto, v. 31, p. 177-196, Jan./Dec.. 1986.

SEITZ, A. Die Gross-Schmeterlinge der Erde. Abteilung II - Die Gross-Scheterlinge des Amerikanischen. 1919-1944. Faunengebietes. Band 7: Eulenartige Nachtfalter. Stuttgart, Alfred Kernen, 96 pranchas, $508 \mathrm{p}$.

SILVA, A. G. A. Mariposa violácea. Nova praga contra o eucalipto. Como se desenvolvem os primeiros combates. Agricultura e pecuária, Rio de Janeiro, v. 20, n. 10, out. 1949.

SILVEIRA NETO, S. et al. Manual de ecologia dos insetos. São Paulo: Ceres, 1976. 419 p.

SILVEIRA NETO, S. et al. Uso da análise faunística de insetos na avaliação do impacto ambiental. Scientia Agrícola, Piracicaba, v. 52, n. 1, p. 9-15, jan./abr. 1995.

TARRAGÓ, M. F. S.; CARVALHO, S.; LINK, D. Levantamento da família Noctuidae, através de armadilha luminosa, em Santa Maria, RS. Revista Centro de Ciências Naturais, Santa Maria, v. 5, n. 2, p. 125-130, dez. 1975.

VIANA, T. M. B.; COSTA, E. C. Lepidópteros associados a duas comunidades florestais em Itaara, RS. Ciência Florestal, Santa Maria, v. 11, n. 1, p. 67-80, jun. 2001.

ZANUNCIO, J. C. et al. Levantamento e flutuação de lepidópteros associados à eucaliptocultura: Região de Montes Claros, Minas Gerais, maio de 1988 a abril de 1989. Revista Ceres, Viçosa, v. 38, n. 218, p. 323- 331, fev./mar. 1991.

ZANUNCIO, J. C. et al. Levantamento e flutuação populacional de lepidópteros associados à eucaliptocultura: VI - Região de Belo Oriente, Minas Gerais. Pesquisa Agropecuária Brasileira, Brasília, v. 28, n. 10, p. 1121-7, out. 1993. 